\title{
Position-Sensitive Fast-Neutron Detector Development in Support of Fuel-Cycle R\&D MPACT Campaign
}

September 2010

Prepared by

P. A. Hausladen

M. A. Blackston

R. J. Newby

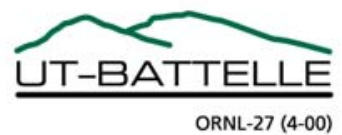




\title{
DOCUMENT AVAILABILITY
}

Reports produced after January 1, 1996, are generally available free via the U.S. Department of Energy (DOE) Information Bridge.

Web site http://www.osti.gov/bridge

Reports produced before January 1, 1996, may be purchased by members of the public from the following source.

\author{
National Technical Information Service \\ 5285 Port Royal Road \\ Springfield, VA 22161 \\ Telephone 703-605-6000 (1-800-553-6847) \\ TDD 703-487-4639 \\ Fax 703-605-6900 \\ E-mail info@ntis.gov \\ Web site http://www.ntis.gov/support/ordernowabout.htm
}

Reports are available to DOE employees, DOE contractors, Energy Technology Data Exchange (ETDE) representatives, and International Nuclear Information System (INIS) representatives from the following source.

Office of Scientific and Technical Information

P.O. Box 62

Oak Ridge, TN 37831

Telephone 865-576-8401

Fax 865-576-5728

E-mail reports@osti.gov

Web site http://www.osti.gov/contact.html

This report was prepared as an account of work sponsored by an agency of the United States Government. Neither the United States Government nor any agency thereof, nor any of their employees, makes any warranty, express or implied, or assumes any legal liability or responsibility for the accuracy, completeness, or usefulness of any information, apparatus, product, or process disclosed, or represents that its use would not infringe privately owned rights. Reference herein to any specific commercial product, process, or service by trade name, trademark, manufacturer, or otherwise, does not necessarily constitute or imply its endorsement, recommendation, or favoring by the United States Government or any agency thereof. The views and opinions of authors expressed herein do not necessarily state or reflect those of the United States Government or any agency thereof. 
Global Nuclear Security Technology Division

\title{
POSITION-SENSITIVE FAST-NEUTRON DETECTOR DEVELOPMENT IN SUPPORT OF FUEL-CYCLE R\&D MPACT CAMPAIGN
}

\author{
P. A. Hausladen, M. A. Blackston, and R. J. Newby
}

Date Published: September 2010

Prepared by

OAK RIDGE NATIONAL LABORATORY

Oak Ridge, Tennessee 37831-6283

managed by

UT-BATTELLE, LLC

for the

U.S. DEPARTMENT OF ENERGY 


\section{CONTENTS}

Page

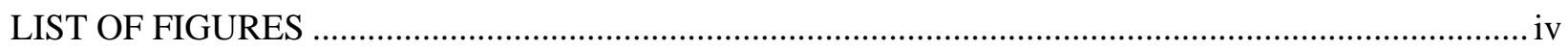

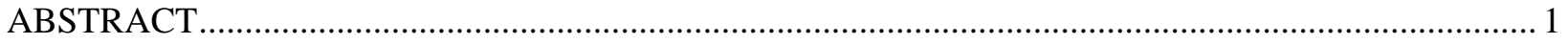

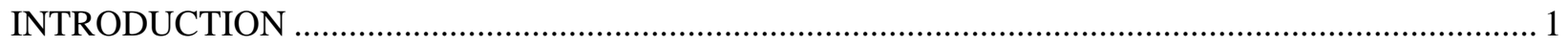

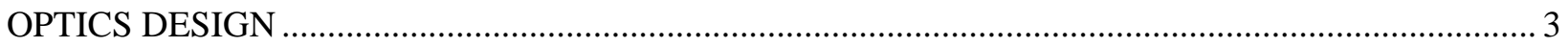

COMPONENT TESTING IN THE RECONFIGURABLE PROTOTYPE .......................................... 4

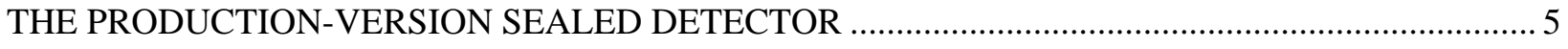

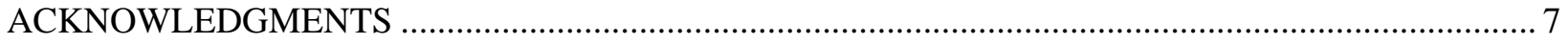

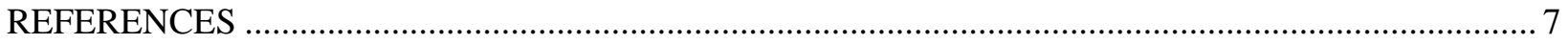




\section{LIST OF FIGURES}

Figure

Page

1 Example configurations of Pu MOX fuel pins and corresponding fast-neutron images ...........1

2 Side and end views of a pixelated plastic scintillator detector and its position response ..........2

3 Conceptual diagram of a "wine rack" reflector grid used to pixelate the liquid volume...........2

4 The optimized light guide geometry and simulated response...........................................4

5 The reconfigurable prototype along with (center) the prototype reflector grid and (right) the reflector grid in the scintillator chamber

6 Position and pulse-shape response of the reconfigurable prototype for (left) the simulated position determination, (center) the measured position determination, and (right) the measured pulse-shape response of the single pixel indicated on the measured position response

7 (Left) production-version sealed detector assembly prior to placement of the mu-metal shield and (right) reflector grid and scintillator chamber prior to installation of the light guide

8 Flood image for partially filled detector showing (left) good position response in corners and (right) reasonable pulse-shape discrimination 


\begin{abstract}
In the present work, fast-neutron imaging detectors with centimeter position resolution are developed whose active volume is liquid scintillator EJ-309, allowing neutron-gamma discrimination via pulse shape. Furthermore, a production version sealed detector is constructed that allows arrangement in a close-packed array for imaging applications. The combination of position resolution, neutron-gamma discrimination, and ready assembly into an imaging array will enable sensitive diagnostic fast-neutron imaging of neutron sources of interest to fuel cycle applications.
\end{abstract}

\title{
INTRODUCTION
}

This report documents results from ORNL development of fast-neutron imaging detectors performed as part of Fuel Cycle R\&D (FCRD) MPACT Campaign work package OR1015040213, “Neutron Interrogation.” In previous work, the authors have demonstrated fastneutron coded-aperture imaging using pixelated detectors constructed from neutron-sensitive plastic scintillator [1]. In these measurements, sensitivity primarily to neutrons was achieved by using a combination of substantial lead shielding, which shielded the detectors from most gamma rays but allowed most neutrons to pass, and a polyethylene mask that modulates fast neutrons but poorly modulates gamma rays. Example images from these measurements, performed at the Zero Power Physics Reactor facility at Idaho National Laboratory with configurations of plutonium mixed-oxide (Pu MOX) fuel, are shown in Figure 1. Included in the figure are photographs of two configurations and the corresponding neutron images: at left, a "clamshell” containing nine 3/8 in. by 6 in. Type 129 fuel pins arranged three deep in the form of an "I" and, at right, a "soup can" containing 90 fuel pins hidden behind a concrete block wall imaged from across the room.
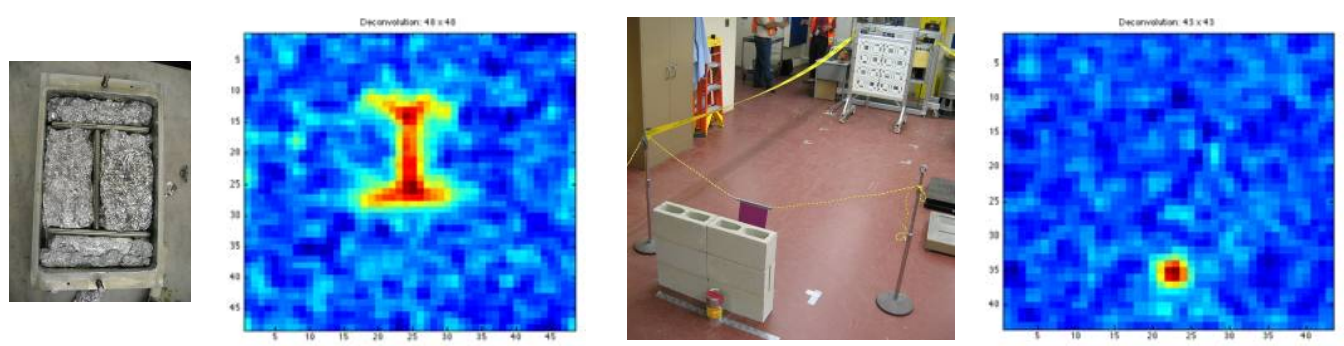

Figure 1. Example configurations of Pu MOX fuel pins and corresponding fast-neutron images.

While these measurements were useful as proof-of-concept demonstrations, the signal-to-noise ratio of the images was limited by the large gamma-ray rate in the detectors. For example, in coded-aperture imaging, the time to reach a desired signal-to-noise in the image scales as the total count rate in the detector divided by the square of the (modulated) signal rate. In most neutron imaging applications, the total count rate in the detectors is dominated by the gamma-ray rate, so eliminating the gamma-ray rate via pulse-shape discrimination prior to imaging will reduce the time required to perform the imaging by roughly the ratio of neutrons to gamma rays. Consequently, a neutron image where the neutrons represent $1 \%$ of the rate in the detectors can be performed 100 times faster with neutron-gamma discrimination in the detectors. In addition, neutron-gamma discrimination eliminates ambiguity from images having a combination of weak 
neutron sources and strong gamma-ray sources by separately imaging gamma rays and neutrons.

In the proof-of-concept imager, the construction of the imaging detectors was similar to that of gamma-ray detectors in conventional positron emission tomography (PET) systems used in medical imaging. Like in PET, each detector consists of an array of pixels separated by reflector and viewed through a segmented light guide by four photomultiplier tubes (PMTs), but in order to be neutron sensitive, the pixel array for the neutron imager was constructed of plastic scintillator. One such detector, along with its position response, is shown in Figure 2.
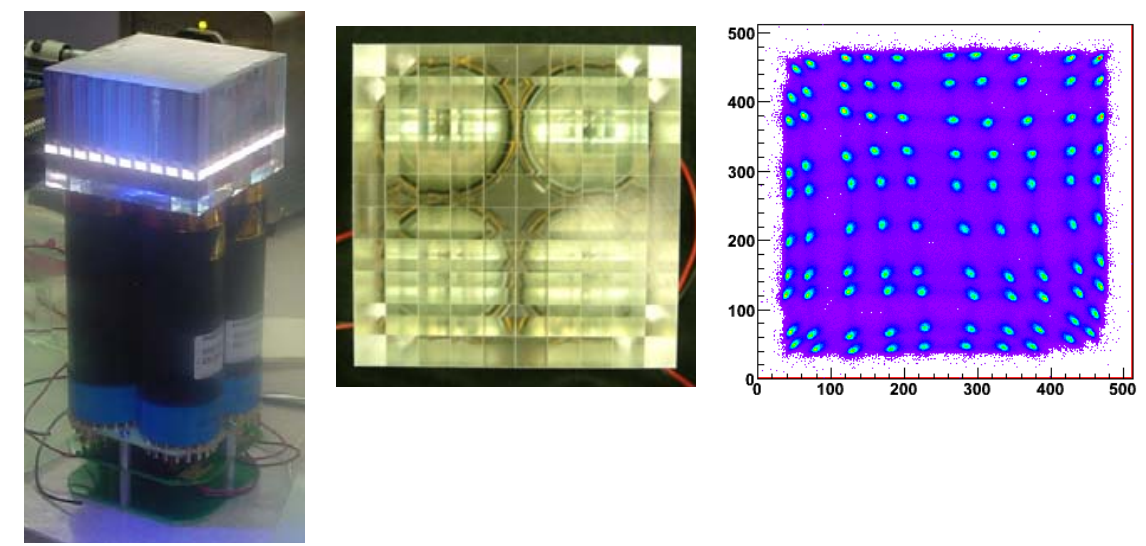

Figure 2. (Left) side and (center) end views of a pixelated plastic scintillator detector and (right) its position response.

In the present work, the intent was to replace the plastic scintillator by liquid scintillator to enable neutron-gamma discrimination via pulse shape. Since an array of 100 individual liquid scintillator capsules would be cost prohibitive, the chosen approach was to submerge a reflector grid (shown conceptually in Figure 3) into a common liquid volume, thus pixelating the volume.

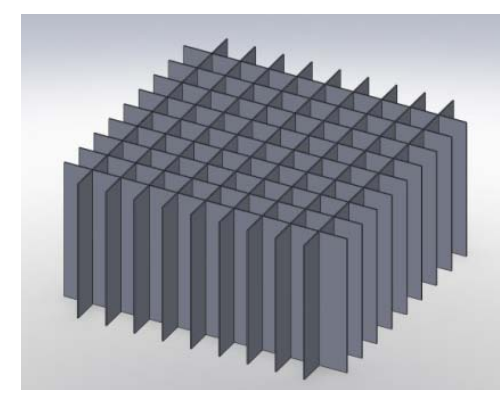

Figure 3. Conceptual diagram of a "wine rack" reflector grid used to pixelate the liquid volume.

The use of liquid scintillator brings with it a number of technical challenges associated with the solvent action of the liquid scintillator and its thermal expansion and contraction. For example, the adhesives and reflector used in the grid need to be impervious to the scintillator's solvent. Furthermore, because the light guide seals the scintillator chamber, it is desirable to have an unbroken surface for the light guide so that the scintillator cannot come in direct contact with adhesive bonds along optical segmentation lines in the light guide. Also, unlike single-volume detectors that normally incorporate an inert-gas bubble to accommodate expansion, a pixelated detector such as this one requires a separate expansion volume so that each pixel always remains filled with scintillator. Connecting the expansion volume with the main detector chamber is 
challenging to accomplish while preserving good light collection for all pixels and introducting minimal dead area into the detector design.

\section{OPTICS DESIGN}

The block detector consists of an array of pixels, a segmented light guide, and a four-PMT readout. The individual pixels in the pixel array are optically isolated $1 \times 1 \times 5 \mathrm{~cm}^{3}$ volumes of scintillator. Because of the large length compared to transverse dimension of the pixels, they also function as a light pipe, where scintillation light acts on average as if it is emitted from the center of the open end facing the PMTs. This light is allowed to spread out in the light guide, which serves as the window of the liquid cell and as an optical connection to the four-PMT readout. The position of the scintillation event within the pixel array is calculated by Anger logic from the amount of light deposited in each PMT. The amount of light sharing among the PMTs is controlled by the light guide, both by its overall length and the optical segmentation within it. For the present work, the design of the light guide was optimized to maximize pixel separation and linearity of response across the detector consistent with (1) the indices of refraction of the scintillator and adhesives to be used in the detector, (2) the presence of a thin layer of unsegmented acrylic to protect the adhesive bonds of the light guide from solvent action, and (3) a minimum number of segments in the light guide to minimize both cost and complexity. In addition, this work provided an opportunity to improve on the response of the previous block detector, seen in Figure 2, where the corner pixels were not as well separated as the pixels in the center of the detector.

During design optimization, the response of potential light guide designs was simulated using the GEANT4 toolkit [3]. In these simulations, each scintillation event in the detector consisted of 200 scintillation photons being emitted isotropically from a point chosen at random within the active volume. The scintillation photons were tracked in the pixel array and light guide until they were either absorbed or detected by a PMT. The inferred $x, y$ position was then calculated from the fraction of light deposited in the rightmost and upper PMTs, respectively, when viewed head-on. The inferred position of many thousands of scintillation events built up a "flood image" (such as that shown at right in Figure 4) where the simulated performance of a design could be evaluated for average pixel separation and linearity of response across the detector to uniform illumination by neutrons.

The ability of the light transport simulations to characterize the performance of a light guide design was checked against experimental results from the previous light guide design for the plastic scintillation detectors. Following validation of the simulation, the light guide design was parameterized in terms of its overall length and the position and depth of internal reflectors within the light guide. The minimum number of internal divisions needed to achieve a nearlinear response was four in each plane, resulting in a light guide constructed from 25 pieces. The outer of these divisions was assumed to span the full depth of the light guide (with the exception of the thin protective layer on the liquid side) and varied in position. Both the position and depth of the second internal reflector were varied. Several hundred configurations were simulated in order to identify an optimized design in terms of pixel separation and linearity of response across the detector. A schematic diagram and a flood image of the optimized design are shown in 
Figure 4.
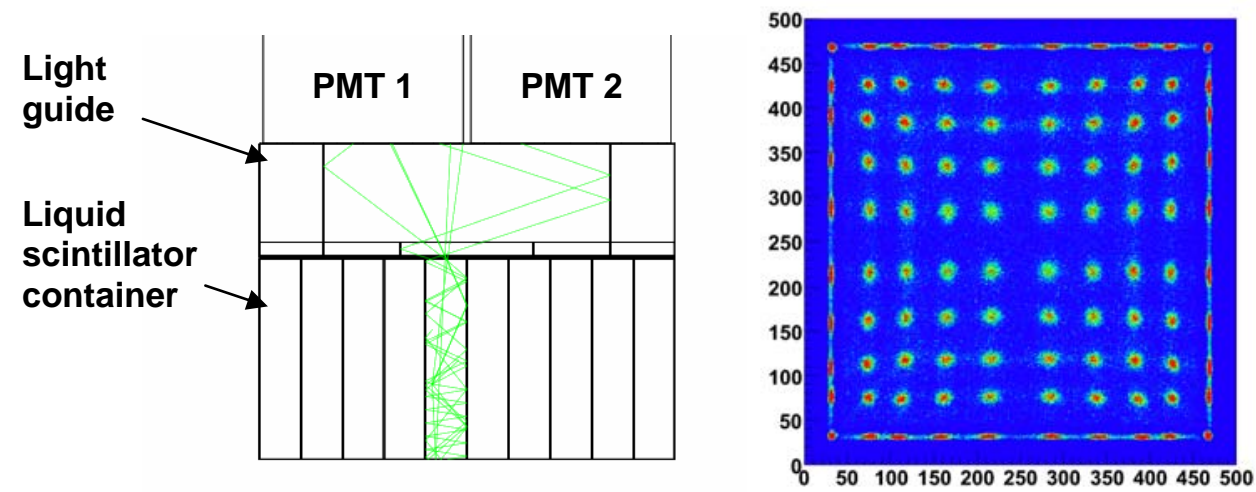

Figure 4. The optimized light guide geometry and simulated response. The vertical lines in the light guide represent reflector boundaries.

\section{COMPONENT TESTING IN THE RECONFIGURABLE PROTOTYPE}

Because of the substantial engineering challenges associated with building a sealed pixelated detector suitable for close-packing in an array, the optical components (including the reflector grid, light guide, and PMTs) were tested in a reconfigurable prototype. This allowed individual components to be modified and returned for testing without the need to manufacture a new detector. The reconfigurable prototype consisted of an open scintillator container into which the reflector grid was inserted, the light guide and PMTs along with a fixture to align them, and a light-tight box with appropriate hookups so that inert gas could be flowed through the box when negative high voltage was applied to the PMTs. The reconfigurable prototype is shown with the door open on the light-tight box in the leftmost panel of Figure 5.

For ease of prototyping, the reflector grid was constructed of $0.5 \mathrm{~mm}$ thick acrylic with 3M Vikuiti reflector bonded to both sides. Testing by the manufacturer of the EJ-309 high-flashpoint liquid scintillator over the course of 9 months indicated that this reflector was inert to solvent action by this scintillator. Furthermore, this is the same high-reflectivity reflector used in the plastic scintillator pixel blocks and light guide. The reflector grid is shown alone in the central panel of Figure 5 and shown inserted in the scintillator container in the rightmost panel.
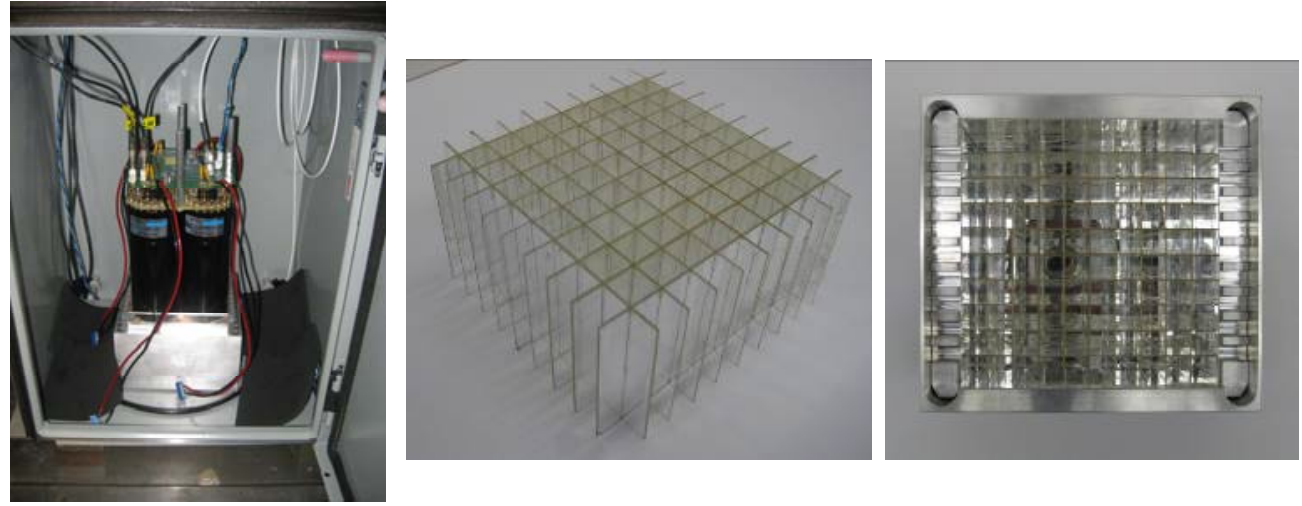

Figure 5. The reconfigurable prototype along with (center) the prototype reflector grid and (right) the reflector grid in the scintillator chamber. 
Testing of the reconfigurable prototype was performed with an AmBe neutron and gamma-ray source, and measured data were used to reconstruct position and pulse shape. Figure 6 shows the simulated position response and measured position response of the detector along with the measured pulse shape for a single pixel. With the exception of a normal level of imperfection expected for any measured position response, the measured position response agrees well with the simulated response, separates all 100 pixels, and has good linearity of response across the entire face of the detector. The pulse-shape measurement, shown in the rightmost panel of Figure 6, indicates a level of neutron-gamma discrimination that is encouraging since the scintillator is exposed to oxygen when poured into the container that holds the reflective grid. Oxygen is a poison for excitation of the slow component of the scintillation light that yields the pulse-shape differences, and the manufacturer of the scintillator indicated that this level of exposure could easily lessen the observed discrimination by a factor of two.
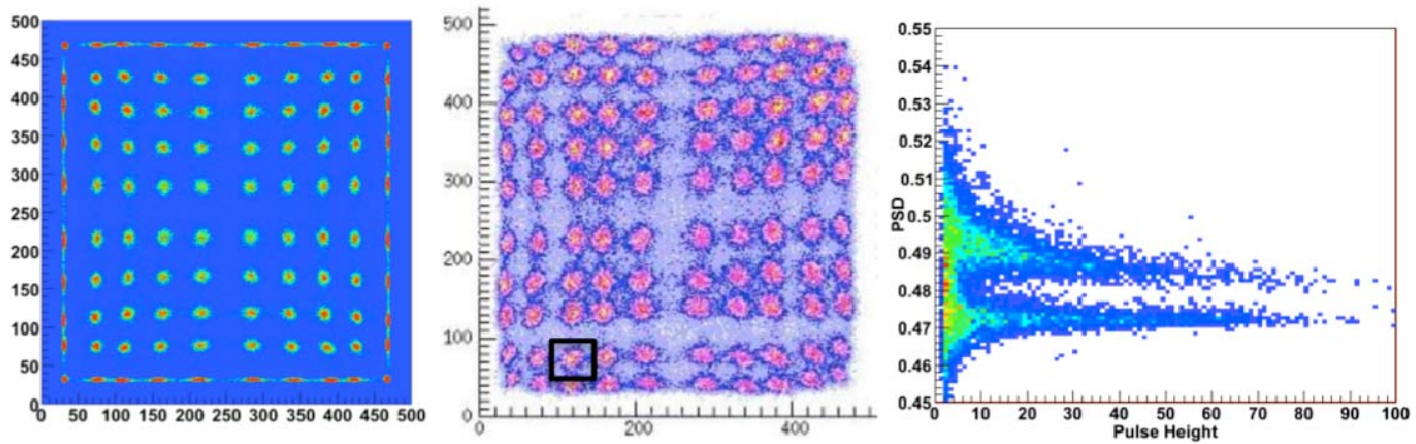

Figure 6. Position and pulse-shape response of the reconfigurable prototype for (left) the simulated position determination, (center) the measured position determination, and (right) the measured pulse-shape response of the single pixel indicated on the measured position response.

With the functioning of the individual components verified in the reconfigurable prototype, construction of a sealed detector that was suitable for packing in an imaging array was undertaken.

\section{THE PRODUCTION-VERSION SEALED DETECTOR}

Procurement of a production-version sealed detector that could be close-packed in an imaging array proved more difficult than originally anticipated, primarily because the traditional vendors for liquid scintillator and liquid scintillator detectors declined to bid on the detector. However, Agile Engineering, that had previously built the optical components (including the reflector grid and light guide) of the reconfigurable prototype and the light guides and pixel blocks for the previous plastic scintillator detectors, was willing to build it. In the production-version detector, the form factor of the previous plastic scintillator detectors was preserved, including the 4.09 in. dimension of the active volume and the 4.25 in. outer dimension of the container. The internal corners of the scintillator container are nearly square to preserve the pixel size of the corner pixels, as can be seen in the right-hand panel of Figure 7. The tolerances on the container necessitated bringing out a conduit to the expansion volume through the light guide. The tubing exiting the light guide and connecting with the expansion volume can be seen in the bottom left corner of Figure 7. Testing with a series of holes drilled in the light guide of the reconfigurable 
prototype indicated that light transmission through a hole in the light guide wetted by scintillator was adequate provided there was no heat damage to the surrounding light guide from the drilling of the hole. As a change from the prototype reflector grid, the structural pieces of the grid for the production detector were manufactured from stainless steel to achieve better tolerances and eliminate warping that took place when curing adhesive on the acrylic prototype. A photograph of the stainless steel grid can be seen at right in Figure 7. Delivery of the production-version sealed detector was accepted by ORNL at the end of August 2010.

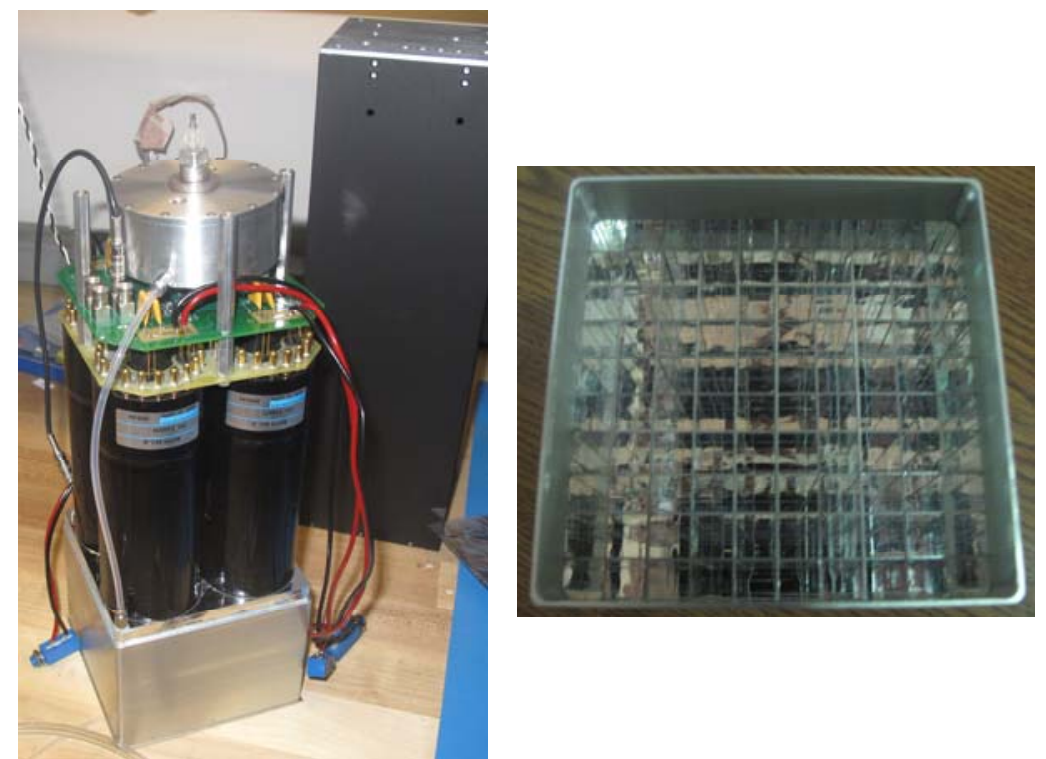

Figure 7. (Left) production-version sealed detector assembly prior to placement of the mu-metal shield and (right) reflector grid and scintillator chamber prior to installation of the light guide. Note the nearly square internal corners of the scintillator chamber.

Initial testing of the detector indicated that the detector volume was incompletely filled with scintillator. Because the tolerances of the grid fit into the scintillator container were poorest along edges and at the box corners, the filling process filled the edge and corner pixels prior to the interior pixels. However, once the path between entry to and exit from the scintillator chamber was filled with scintillator, the interior pixels remained empty. The detector has been returned to the vendor to drain and evacuate the scintillator chamber prior to refilling. In addition, future grids will be manufactured with more pronounced channels to enable improved flow of scintillator during the filling process.

Despite partial filling of the scintillator chamber, the performance of the detector could be characterized for the edge and corner pixels of the detector, where performance of detectors is typically the worst. Figure 8 shows a flood image for the partially filled detector showing excellent pixel separation along the edges and corners. In particular, the corner with the feedthrough to the expansion chamber cannot be readily distinguished from the other corners. The performance of the detector will be more thoroughly tested upon its return from the vendor. 

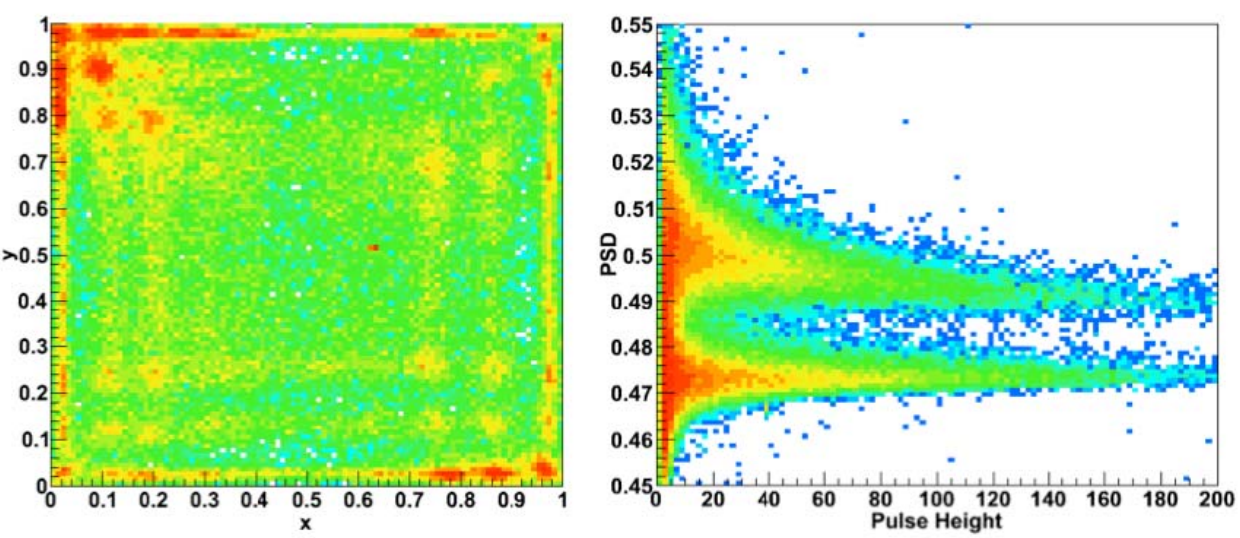

Figure 8. Flood image for partially filled detector showing (left) good position response in corners and (right) reasonable pulse-shape discrimination.

\section{ACKNOWLEDGMENTS}

The authors gratefully acknowledge Charles Hurlbut of Eljen Technology, for instructive suggestions and testing of reflector and adhesives for durability in the EJ-309 solvent, and Agile Engineering, for construction of the detector optics and development of the production detector.

\section{REFERENCES}

[1] P.A. Hausladen and M.A. Blackston, Passive and Active Fast-Neutron Imaging in Support of AFCI Safeguards Campaign, report ORNL/TM-2009/210, Oak Ridge National Laboratory, 2009.

[2] H. Frezghi, M.A. Blackston, P.A. Hausladen, and L. Fabris, IEEE Nuclear Science Symposium Conference Record, pp. 3128 -3132, 19-25 October 2008.

[3] J. Allison et al., IEEE Trans. Nucl. Sci., vol. 53, no. 1, pp. 270-278, February 2006. 